\title{
Amplify-and-Forward Relay Selection with Outdated Channel State Information
}

\author{
Diomidis S. Michalopoulos*, Himal A. Suraweera ${ }^{\dagger}$, George K. Karagiannidis ${ }^{\ddagger}$, and Robert Schober* \\ * Department of Electrical \& Computer Engineering, The University of British Columbia, Vancouver, BC, V6T 1Z4, Canada \\ $\dagger^{\dagger}$ Department of Electrical \& Computer Engineering, National University of Singapore, Singapore 119260 \\ ${ }_{\ddagger}^{\ddagger}$ Department of Electrical \& Computer Engineering, Aristotle University of Thessaloniki, GR-54124, Thessaloniki, Greece
}

\begin{abstract}
We study the effect of outdated channel estimation on the outage performance of amplify-and-forward (AF) relay selection, where only one out of the set of available relays is activated. In particular, we derive closed-form expressions for the outage probability of two variations of $\mathrm{AF}$ relay selection, namely best relay selection and partial relay selection, when the selection is based upon outdated channel estimates. Numerical results manifest that the outage performance of both schemes under consideration is highly dependent on the level of imperfection of the channel estimates. It is further shown that it may be preferable, in terms of outage probability, not to include links in the relay selection process that experience high maximum Doppler shifts.
\end{abstract}

\section{INTRODUCTION}

Various cooperative relay schemes have been explored in the literature because their deployment in wireless networks has the potential of offering a number of significant performance benefits, including hotspot throughput improvements and cellular signal coverage enhancements [1]. As a result, future mobile broadband communication networks such as 3GPP LTEAdvanced, IEEE $802.16 \mathrm{j}$, and IEEE $802.16 \mathrm{~m}$ are expected to support relay based communication [2]. Relays in wireless networks can be classified as decode-and-forward (DF), where the relay decodes and sometimes re-encodes the information before forwarding it, and amplify-and-forward (AF), where the relay forwards the signal without hard decoding.

The advantages of cooperative diversity come at the expense of a loss in spectral efficiency since the source and all the relays must transmit in orthogonal channels [3]. The inefficient utilization of the channel resources can be mitigated by using relay selection. One such scheme is the best relay protocol proposed in [4]. In this scheme, a single best relay is selected to retransmit to the destination. Hence, only two orthogonal channels (regardless of the number of relays) are required in this case. It was also shown in [4] that by selecting the relay with the best end-to-end path between the source and the destination, a diversity gain on the order of the number of relays in the network can be realized. However, in some applications such as resource-constrained ad hoc and sensor networks, monitoring the connectivity among all links can limit the network lifetime. Such challenges have motivated the development of partial relay selection schemes, which require channel state information (CSI) of only the source-relay links, or alternatively, only the relay-destination links [5].

Despite the rich body of literature on relay selection schemes (see, e.g., [4]-[11] and the references therein), the performance of best AF relay selection considering outdated CSI has not been investigated, to the best of the authors' knowledge. Nevertheless, the outdated CSI case is of significant interest because the CSI is rarely perfect in practice. To this end, in this paper we provide closed-form expressions for the outage probability for the AF best relay selection and the partial relay selection schemes, described above. Such analysis leads to interesting numerical results, which indicate that the performance of relay selection is highly dependent on the available knowledge regarding the CSI of the participating links. In addition, it is shown that partial relay selection may outperform best relay selection in cases where the maximum Doppler frequency in either the source-relay or the relay-destination links is higher than a certain threshold.

\section{SySTEM MODEL}

We consider the cooperative relaying setup illustrated in Fig. 1 , which consists of a single source terminal $S, N$ relays denoted by $R_{i}, i=1, \ldots, N$, and a single destination terminal, $D$. All relays operate in the half-duplex amplify-and-forward (AF) mode; they also employ the so-called CSI-assisted variable gain, where the relaying gain depends on the instantaneous channel amplitude of the corresponding $S-R_{i}$ link. The fading in all $S-R_{i}$ and $R_{i}-D$ paths is assumed independent and identically distributed according to the Rayleigh distribution. In addition, no direct $S-D$ link is assumed, hence the communication between $S$ and $D$ is implemented only via the relaying terminals. We denote the circularly symmetric complex Gaussian channel gain between nodes $A$ and $B$ as $h_{A B}$; we use the notation $\gamma_{A B}$ to refer to the instantaneous signal-tonoise-ratio (SNR) of link $A-B$, so that $\gamma_{A B}=\left|h_{A B}\right|^{2} / N_{0}$, where we have assumed for simplicity that all nodes transmit with unit power and $N_{0}$ represents the additive white Gaussian noise (AWGN) power. Moreover, we use the notations $f_{X}(\cdot)$ and $F_{X}(\cdot)$ to refer to the probability density function (pdf) and the cumulative density function (cdf) of random variable (RV) $X$, respectively.

Among the $N$ available relays only a single relay, as selected through a procedure based on the highest instantaneous SNR, is supposed to forward the signal transmitted by source, $S$, to destination, $D$. Selecting the "best" relay from the set of available ones results in a somewhat opportunistic usage of the relays, as also pointed out in [4]. The decision on the selected relay is assumed to be made in a central unit (CU), which collects all the information regarding the instantaneous channel 


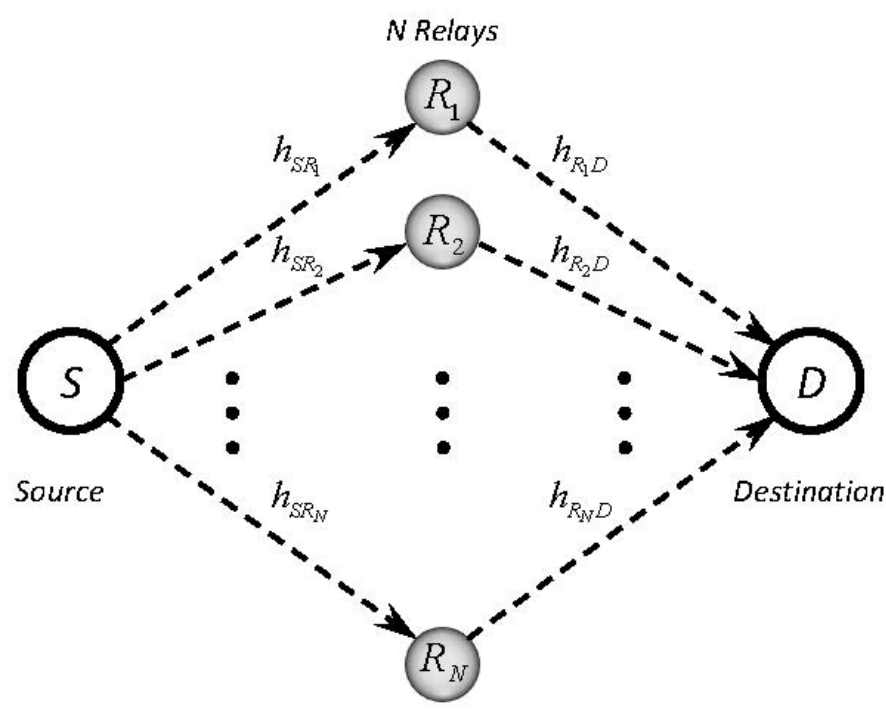

Fig. 1. Model of the system under consideration.

states and feeds back the result of the selection process to the relays.

In contrast to the majority of works on CSI-assisted $\mathrm{AF}$ relays, in this paper, we assume that the CU has outdated CSI, in the sense that the decision regarding the "best" relay does not correspond to the current time instance because of, e.g., feedback delay. Therefore, denoting by $\hat{h}_{S R_{i}}$ and $\hat{h}_{R_{i} D}$ the partially known channels of the $S-R_{i}$ and $R_{i}-D$ links at the time of selection, we model the outdated CSI that the CU has for the $i$ th relay as [12], [13]

$$
\hat{h}_{S R_{i}}=\rho_{1} h_{S R_{i}}+\sqrt{1-\rho_{1}^{2}} w_{S R_{i}}
$$

and

$$
\hat{h}_{R_{i} D}=\rho_{2} h_{R_{i} D}+\sqrt{1-\rho_{2}^{2}} w_{R_{i} D}
$$

where $w_{S R_{i}}$ and $w_{R_{i} D}$ are circularly symmetric complex Gaussian RVs having the same variance as $\mathrm{RVs} h_{S R_{i}}$ and $h_{R_{i} D}$, respectively; $\rho_{1}\left(\rho_{2}\right)$ is the correlation coefficient between $h_{S R_{i}}$ and $\hat{h}_{S R_{i}}\left(h_{R_{i} D}\right.$ and $\left.\hat{h}_{R_{i} D}\right)$, which using the Jakes' autocorrelation model is given by

$$
\begin{aligned}
& \rho_{1}=J_{0}\left(2 \pi f_{d, S R} T_{d}\right) \\
& \rho_{2}=J_{0}\left(2 \pi f_{d, R D} T_{d}\right)
\end{aligned}
$$

with $J_{0}(\cdot)$ denoting the Bessel function of the first kind of order zero [14, Eq. (8.411)]; $f_{d, A B}$ is the maximum Doppler frequency on the $A-B$ link and $T_{d}$ is the time difference between the actual channel value and its estimate. Due to the CSI-assisted AF mode of operation, the end-to-end SNR for the $i$ th relay, $\gamma_{i}, i=1, \ldots, N$, can be expressed as [3]

$$
\gamma_{i}=\frac{\gamma_{S R_{i}} \gamma_{R_{i} D}}{\gamma_{S R_{i}}+\gamma_{R_{i} D}+1}
$$

where we have assumed that the CSI at the relay and the destination is perfect, regarding the selected path; in other words, although there exists outdated CSI at the CU, this outdated CSI does not affect the detection process at the destination, nor the CSI-assisted gain posed by the activated relay.

It is further assumed that there exists symmetry among the $S-R_{i}$ and $R_{i}-D$ links, in the sense that the fading on the $S-R_{i}$ links is independent and identically distributed (i.i.d.), so that the average SNR in all the $S-R_{i}$ links is given by $\bar{\gamma}_{S R}$; the $R_{i}-D$ links are also assumed to be i.i.d., with average SNR denoted by $\bar{\gamma}_{R D}$. Hence, due to symmetry, the pdfs of $\gamma_{S R_{i}}$ and $\gamma_{R_{i} D}, i=1, \ldots, N$, are denoted by $f_{\gamma_{S R}}(\cdot)$ and $f_{\gamma_{R D}}(\cdot)$, respectively; the cdfs of $\gamma_{S R_{i}}$ and $\gamma_{R_{i} D}$ are likewise denoted by $F_{\gamma_{S R}}(\cdot)$ and $F_{\gamma_{R D}}(\cdot)$, respectively. Moreover, we assume that the direct $S-D$ channel is weak enough, as compared to the selected relaying path, so as to be considered negligible.

\section{A. Best Relay Selection}

In this paper we use the term "best relay selection" to refer to the case where the decision on the selected relay is determined by both the $S-R_{i}$ and $R_{i}-D$ links. In particular, we assume that the relay with the strongest "bottleneck" link is selected. Hence, the end-to-end SNR that the CU assumes based upon an outdated estimation is given by [4]

$$
\hat{\gamma}_{i}=\min \left(\hat{\gamma}_{S R_{i}}, \hat{\gamma}_{R_{i} D}\right)
$$

where $\hat{\gamma}_{A B}$ denotes the estimate of $\gamma_{A B}$. The CU thus activates the relay that satisfies the following condition

$$
k=\arg \max _{i}\left(\hat{\gamma}_{i}\right) .
$$

\section{B. Partial Relay Selection}

In cases where the $\mathrm{CU}$ has information only of either the $S-R_{i}$ or the $R_{i}-D$ path, the selection procedure is modified accordingly so that only one of the above links is taken into account. In the sequel, partial relay selection will refer to the case where only the $S-R_{i}$ CSI is available; the selected relay is thus determined by [5]

$$
k=\arg \max _{i}\left(\hat{\gamma}_{S R_{i}}\right) .
$$

Apparently, the analysis can be easily extended to the case where only $R_{i}-D$ CSI is available.

\section{Outage Probability}

The outage probability is defined as the probability that the system cannot support a target data rate, which is equivalent to the probability that the overall SNR is lower than a threshold, $\gamma_{T}$; this threshold is related to the target rate $r$ through $\gamma_{T}=$ $2^{2 r}-1$. Consequently, we may express the outage probability as

$$
\operatorname{Pr}\left(\gamma_{k}<\gamma_{T}\right)=F_{\gamma_{k}}\left(\gamma_{T}\right) .
$$

In order to obtain the system's outage probability, we need to find the cdf $F_{\gamma_{k}}(\gamma)$. We proceed further and express this cdf as

$$
\begin{aligned}
F_{\gamma_{k}}\left(\gamma_{T}\right) & =\operatorname{Pr}\left(\frac{\gamma_{S R_{k}} \gamma_{R_{k} D}}{\gamma_{S R_{k}}+\gamma_{R_{k} D}+1}<\gamma_{T}\right) \\
& =\int_{0}^{\infty} \operatorname{Pr}\left(\frac{\gamma_{S R_{k}} y}{\gamma_{S R_{k}}+y+1}<\gamma_{T}\right) f_{\gamma_{R_{k} D}}(y) d y .
\end{aligned}
$$


Splitting the integration interval in (9) into $\left[0, \gamma_{T}\right)$ and $\left[\gamma_{T}, \infty\right)$ and after applying some algebraic manipulations, $F_{\gamma_{k}}\left(\gamma_{T}\right)$ can be expressed as

$$
\begin{aligned}
F_{\gamma_{k}}\left(\gamma_{T}\right)= & \int_{0}^{\gamma_{T}} f_{\gamma_{R_{k} D}}(y) d y \\
& +\int_{\gamma_{T}}^{\infty} F_{\gamma_{S R_{k}}}\left(\frac{\gamma_{T} y+\gamma_{T}}{y-\gamma_{T}}\right) f_{\gamma_{R_{k} D}}(y) d y
\end{aligned}
$$

Hence, in order to find the outage probability we need expressions for the functions $F_{\gamma_{S R_{k}}}(\cdot)$ and $f_{\gamma_{R_{k}} D}(\cdot)$. In the following two subsections, we derive these expression separately for the best relay selection and the partial relay selection case, respectively.

\section{A. Best Relay Selection}

In this case, the relay selection is as in (7), hence the statistics of the $S-R_{k}$ and $R_{k}-D$ links are determined by both the $S-R_{i}$ and $R_{i}-D, i=1, \ldots, N$, links. Due to the fact that the distributions of $\gamma_{S R_{i}}$ and $\hat{\gamma}_{S R_{i}}$ are correlated exponential distributions, their joint pdf is given by [12]

$$
f_{\gamma_{S R_{i}}, \hat{\gamma}_{S R_{i}}}(x, y)=\frac{e^{-\frac{x+y}{\left(1-\rho_{1}^{2}\right) \bar{\gamma}_{S R}}}}{\left(1-\rho_{1}^{2}\right) \bar{\gamma}_{S R}^{2}} I_{0}\left(\frac{2 \sqrt{\rho_{1}^{2} x y}}{\left(1-\rho_{1}^{2}\right) \bar{\gamma}_{S R}}\right) \text {. }
$$

The pdf of the SNR of the source-relay part of the selected path, $\gamma_{S R_{k}}$, is given by

$$
f_{\gamma_{S R_{k}}}(x)=\int_{0}^{\infty} f_{\gamma_{S R_{k}} \mid \hat{\gamma}_{S R_{k}}}(x \mid y) f_{\hat{\gamma}_{S R_{k}}}(y) d y .
$$

Then, given the fact the $\gamma_{S R_{i}}$, as well as $\hat{\gamma}_{S R_{i}}, i=1, \ldots, N$, are i.i.d. RVs, the conditional pdf $f_{\gamma_{S R_{i}}} \mid \hat{\gamma}_{S R_{i}}(\cdot \mid \cdot)$ is identical for each $i=1, \ldots, N$, and expressed as $f_{\gamma_{S R} \mid \hat{\gamma}_{S R}}(\cdot \cdot)$. Moreover, it follows from the theory of the concomitants of order statistics that also the conditional pdf $f_{\gamma_{S R_{k}} \mid \hat{\gamma}_{S R_{k}}}(\cdot \mid \cdot)$ is identical to $f_{\gamma_{S R} \mid \hat{\gamma}_{S R}}(\cdot \mid \cdot)$ [15], yielding

$$
f_{\gamma_{S R_{k}} \mid \hat{\gamma}_{S R_{k}}}(x \mid y)=\frac{f_{\gamma_{S R}, \hat{\gamma}_{S R}}(x, y)}{f_{\hat{\gamma}_{S R}}(y)}
$$

where $f_{\hat{\gamma}_{S R}}(y)=\frac{1}{\bar{\gamma}_{S R}} e^{-\frac{y}{\bar{\gamma}_{S R}}}$, since the statistics of $\hat{\gamma}_{S R_{i}}$ is the same as that of $\gamma_{S R_{i}}$, because the RVs $h_{S R_{i}}$ and $w_{S R_{i}}$ in (1) share the same statistics. Therefore, substituting (11) into (13) we obtain

$$
f_{\gamma_{S R_{k}} \mid \hat{\gamma}_{S R_{k}}}(x \mid y)=\frac{e^{-\frac{x+\rho_{1}^{2} y}{\left(1-\rho_{1}^{2}\right) \bar{\gamma}_{S R}}}}{\left(1-\rho_{1}^{2}\right) \bar{\gamma}_{S R}} I_{0}\left(\frac{2 \sqrt{\rho_{1}^{2} x y}}{\left(1-\rho_{1}^{2}\right) \bar{\gamma}_{S R}}\right) .
$$

The cdf of $\hat{\gamma}_{S R_{k}}, F_{\hat{\gamma}_{S R_{k}}}(\cdot)$, is derived as follows. Due to symmetry among the $N$ end-to-end paths

$$
\begin{aligned}
F_{\hat{\gamma}_{S R_{k}}}(x) & =N \operatorname{Pr}\left\{\hat{\gamma}_{S R_{i}} \leq x \cap k=i\right\} \\
& =N \int_{0}^{x} f_{\hat{\gamma}_{S R_{i}}}(y) \operatorname{Pr}\left\{k=i \mid \hat{\gamma}_{S R_{i}}=y\right\} d y
\end{aligned}
$$

holds. Considering that the "best" relay is selected according to (6) and (7), it is the weakest of the $S-R_{i}, R_{i}-D$ links, as estimated at the $\mathrm{CU}$, which determines $\hat{\gamma}_{i}, i=1, \ldots, N$. Therefore, we can express the probability $\operatorname{Pr}\left\{k=i \mid \hat{\gamma}_{S R_{i}}=y\right\}$ in (15) as the summation of two mutually exclusive events, corresponding to the cases where $\hat{\gamma}_{S R_{i}}<\hat{\gamma}_{R_{i} D}$ and $\hat{\gamma}_{S R_{i}}<\hat{\gamma}_{R_{i} D}$, yielding

$$
\begin{aligned}
& F_{\hat{\gamma}_{S R_{k}}}(x)= \\
& N\left[\int_{0}^{x} f_{\hat{\gamma}_{S R_{i}}}(y)\left(\int_{0}^{y} f_{\hat{\gamma}_{R_{i} D}}(\omega)\left[F_{\hat{\gamma}_{i}}(\omega)\right]^{N-1} d \omega\right) d y\right. \\
& \left.+\int_{0}^{x} f_{\hat{\gamma}_{S R_{i}}}(y)\left(\int_{y}^{\infty} f_{\hat{\gamma}_{R_{i} D}}(\omega) d \omega\right)\left[F_{\hat{\gamma}_{i}}(y)\right]^{N-1} d y\right]
\end{aligned}
$$

where, because of symmetry, the index $i$ may take any value of the set $\{1, \ldots, N\}$. Given that $\hat{\gamma}_{S R}$ and $\hat{\gamma}_{R D}$ follow the same distribution as $\gamma_{S R}$ and $\gamma_{R D}$, respectively, the cdf of $\hat{\gamma}_{i}$ is derived as $F_{\hat{\gamma}_{i}}(x)=1-\exp (-x / \bar{\gamma})$, where $\bar{\gamma}=$ $\bar{\gamma}_{S R} \bar{\gamma}_{R D} /\left(\bar{\gamma}_{S R}+\bar{\gamma}_{R D}\right)$ [10]. Consequently, we obtain

$$
\left[F_{\hat{\gamma}_{i}}(x)\right]^{N-1}=\sum_{n=0}^{N-1}(-1)^{n}\left(\begin{array}{c}
N-1 \\
n
\end{array}\right) \exp \left(-\frac{n x}{\bar{\gamma}}\right) .
$$

The inner integral in the first line of (16) is evaluated as

$$
\begin{aligned}
& \int_{0}^{y} f_{\hat{\gamma}_{R_{i} D}}(\omega)\left[F_{\hat{\gamma}_{i}}(\omega)\right]^{N-1} d \omega \\
& =\bar{\gamma} \sum_{n=0}^{N-1} \frac{(-1)^{n}}{n \bar{\gamma}_{R D}+\bar{\gamma}}\left(\begin{array}{c}
N-1 \\
n
\end{array}\right)\left(1-e^{-\left(\frac{n}{\bar{\gamma}}+\frac{1}{\gamma_{R D}}\right) y}\right) .
\end{aligned}
$$

Likewise, we may express the first line of (16) as

$$
\begin{aligned}
& \int_{0}^{x} f_{\hat{\gamma}_{S R}}(y)\left(\int_{0}^{y} f_{\hat{\gamma}_{R_{i}}}(\omega)\left[F_{\hat{\gamma}_{i}}(\omega)\right]^{N-1} d \omega\right) d y \\
& =\bar{\gamma} \sum_{n=0}^{N-1} \frac{(-1)^{n}}{n \bar{\gamma}_{R D}+\bar{\gamma}}\left(\begin{array}{c}
N-1 \\
n
\end{array}\right)\left(1-e^{-\frac{x}{\bar{\gamma}_{S R}}}\right) \\
& -\frac{\bar{\gamma}}{\bar{\gamma}_{S R}} \sum_{n=0}^{N-1} \frac{(-1)^{n}\left(\begin{array}{c}
N-1 \\
n
\end{array}\right)}{n \bar{\gamma}_{R D}+\bar{\gamma}} \frac{\left(1-e^{-\left(\frac{n}{\bar{\gamma}}+\frac{1}{\bar{\gamma}_{R D}}+\frac{1}{\bar{\gamma}_{S R}}\right) x}\right)}{\left(\frac{n}{\bar{\gamma}}+\frac{1}{\bar{\gamma}_{R D}}+\frac{1}{\bar{\gamma}_{S R}}\right)}
\end{aligned}
$$

and the second line of (16) as

$$
\begin{aligned}
& \int_{0}^{x} f_{\hat{\gamma}_{S R}}(y)\left(\int_{y}^{\infty} f_{\hat{\gamma}_{R_{i} D}}(\omega) d \omega\right)\left[F_{\hat{\gamma}_{i}}(y)\right]^{N-1} d y \\
& =\frac{1}{\bar{\gamma}_{S R}} \sum_{n=0}^{N-1} \frac{(-1)^{n}\left(\begin{array}{c}
N-1 \\
n
\end{array}\right)\left(1-e^{-\left(\frac{1}{\bar{\gamma}_{S R}}+\frac{1}{\bar{\gamma}_{R D}}+\frac{n}{\bar{\gamma}}\right) x}\right)}{\left(\frac{1}{\bar{\gamma}_{S R}}+\frac{1}{\bar{\gamma}_{R D}}+\frac{n}{\bar{\gamma}}\right)}
\end{aligned}
$$

Hence, combining (16)-(20) we get the cdf of $\hat{\gamma}_{S R_{k}}$ as

$$
\begin{aligned}
& F_{\hat{\gamma}_{S R_{k}}}(x)=N\left[\overline { \gamma } \sum _ { n = 0 } ^ { N - 1 } \frac { ( - 1 ) ^ { n } ( \begin{array} { c } 
{ N - 1 } \\
{ n }
\end{array} ) } { n \overline { \gamma } _ { R D } + \overline { \gamma } } \left(1-e^{\left.-\frac{x}{\bar{\gamma}_{S R}}\right)}\right.\right. \\
& \left.-\frac{\bar{\gamma}}{\bar{\gamma}_{S R}} \sum_{n=0}^{N-1} \frac{(-1)^{n}\left(\begin{array}{c}
N-1 \\
n
\end{array}\right)\left(1-e^{-\left(\frac{n}{\left.\bar{\gamma}+\frac{1}{\bar{\gamma}_{R D}}+\frac{1}{\bar{\gamma}_{S R}}\right) x}\right)}\right.}{\left(n \bar{\gamma}_{R D}+\bar{\gamma}\right)\left(\frac{n}{\bar{\gamma}}+\frac{1}{\bar{\gamma}_{R D}}+\frac{1}{\bar{\gamma}_{S R}}\right)}\right] . \\
& \left.+\frac{1}{\bar{\gamma}_{S R}} \sum_{n=0}^{N-1} \frac{(-1)^{n}\left(\begin{array}{c}
N-1 \\
n
\end{array}\right)\left(1-e^{-\left(\frac{1}{\bar{\gamma}_{S R}}+\frac{1}{\bar{\gamma}_{R D}}+\frac{n}{\bar{\gamma}}\right) x}\right)}{\left(\frac{1}{\bar{\gamma}_{S R}}+\frac{1}{\bar{\gamma}_{R D}}+\frac{n}{\bar{\gamma}}\right)}\right]
\end{aligned}
$$


The pdf of $\hat{\gamma}_{S R_{k}}$ is derived by differentiating (21), yielding

$$
\begin{aligned}
f_{\hat{\gamma}_{S R_{k}}}(x)= & N\left(\begin{array}{c}
N-1 \\
n
\end{array}\right)\left[\frac{\bar{\gamma}(-1)^{n} \exp \left(-\frac{x}{\bar{\gamma}_{S R}}\right)}{\bar{\gamma}_{S R}\left(\bar{\gamma}+n \bar{\gamma}_{R D}\right)}\right. \\
& +\frac{\bar{\gamma}(-1)^{n+1} e^{-\left(\frac{1}{\bar{\gamma}_{S R}}+\frac{1}{\bar{\gamma}_{R D}}+\frac{n}{\bar{\gamma}}\right) x}}{\bar{\gamma}_{S R}\left(\bar{\gamma}+n \bar{\gamma}_{R D}\right)} \\
& \left.+\frac{(-1)^{n} e^{-\left(\frac{1}{\bar{\gamma}_{S R}}+\frac{1}{\bar{\gamma}_{R D}}+\frac{n}{\bar{\gamma}}\right) x}}{\bar{\gamma}_{S R}}\right] .
\end{aligned}
$$

As a result, we may evaluate the pdf of $\gamma_{S R_{k}}$ by substituting (14) and (22) into (12), yielding

$$
\begin{aligned}
& f_{\gamma_{S R_{k}}}(x)=N \sum_{n=0}^{N-1}(-1)^{n}\left(\begin{array}{c}
N-1 \\
n
\end{array}\right)\left[\frac{\exp \left(-\frac{x}{\bar{\gamma}_{S R}}\right)}{\bar{\gamma}_{S R}+n\left(\bar{\gamma}_{S R}+\bar{\gamma}_{R D}\right)}\right. \\
& -\frac{\bar{\gamma}_{R D}}{\bar{\gamma}_{S R}+n\left(\bar{\gamma}_{S R}+\bar{\gamma}_{R D}\right)} \\
& \times \frac{e^{-\frac{(n+1)\left(\bar{\gamma}_{S R}+\bar{\gamma}_{R D}\right) x}{\bar{\gamma}_{S R}(n+1)\left(\bar{\gamma}_{S R}+\bar{\gamma}_{R D}\right)-\bar{\gamma}_{S R} \rho_{1}^{2}\left[\bar{\gamma}_{S R}+n\left(\bar{\gamma}_{S R}+\bar{\gamma}_{R D}\right)\right]}}}{(n+1)\left(\bar{\gamma}_{S R}+\bar{\gamma}_{R D}\right)+\rho_{1}^{2}\left(\bar{\gamma}_{S R}+n\left(\bar{\gamma}_{S R}+\bar{\gamma}_{R D}\right)\right)} \\
& \left.+\frac{\bar{\gamma} \bar{\gamma}_{R D} e^{-\frac{\left(\bar{\gamma}_{S R} \bar{\gamma}_{R D}+n \bar{\gamma}_{S R} \bar{\gamma}_{R D}\right) x}{\bar{\gamma}_{S R}\left[n \bar{\gamma}_{S R} \bar{\gamma}_{R D}\left(1-\rho_{1}^{2}\right)+\bar{\gamma}\left(\bar{\gamma}_{S R} \bar{\gamma}_{R D}-\bar{\gamma}_{S R} \rho_{1}^{2}\right)\right]}}}{\bar{\gamma}_{S R}\left[n\left(\bar{\gamma}_{S R}+\bar{\gamma}_{R D}\right)\left(1-\rho_{1}^{2}\right)+\bar{\gamma}\left(\bar{\gamma}_{S R} \bar{\gamma}_{R D}-\bar{\gamma}_{S R} \rho_{1}^{2}\right)\right]}\right]
\end{aligned}
$$

The cdf of $\gamma_{S R_{k}}$ can be derived directly from (23) as

$$
\begin{aligned}
& F_{\gamma_{S R_{k}}}(z)=N \sum_{n=0}^{N-1} \frac{(-1)^{n}\left(\begin{array}{c}
N-1 \\
n
\end{array}\right) n \bar{\gamma}_{R D}}{(n+1)\left[\bar{\gamma}_{S R}+n\left(\bar{\gamma}_{S R}+\bar{\gamma}_{R D}\right)\right]} \\
& \times\left[1-e^{-\frac{(n+1)\left(\bar{\gamma}_{S R}+\bar{\gamma}_{R D}\right) x}{\bar{\gamma}_{S R}(n+1)\left(\bar{\gamma}_{S R}+\bar{\gamma}_{R D}\right)-\bar{\gamma}_{S R} \rho_{1}^{2}\left[\bar{\gamma}_{S R}+n\left(\bar{\gamma}_{S R}+\bar{\gamma}_{R D}\right)\right]}}\right. \\
& \left.+\frac{\bar{\gamma}_{S R}(n+1)}{n \bar{\gamma}_{R D}}\left(1-\exp \left(-\frac{x}{\bar{\gamma}_{S R}}\right)\right)\right]
\end{aligned}
$$

Furthermore, the pdf of $\gamma_{R_{k} D}$ is derived from (23), due to symmetry, by mutually interchanging $\bar{\gamma}_{S R}$ and $\bar{\gamma}_{R D}$, while substituting $\rho_{1}$ with $\rho_{2}$. Hence, the outage probability of best relay selection is derived by substituting (24) and $f_{\gamma_{R_{k} D}}(\cdot)$, as taken from (23), into (10). Then, applying the change of variables, $\omega=y-\gamma_{T}$, and making use of the integral $\int_{0}^{\infty} \exp (-a x) \exp \left(-\frac{\kappa x+c}{\lambda x}\right) d x=$ $2 \sqrt{c /(a \lambda)} \exp (-\kappa / \lambda) K_{1}(2 \sqrt{a c / \lambda})[14, \quad$ Eq. (3.324.1)], where $K_{1}(\cdot)$ is the first order modified Bessel function of the second kind, the outage probability is derived as shown in (25) given at the top of the next page. One may also note that, despite its length, (25) can be readily evaluated since it involves simple algebraic calculations, while the function $K_{1}(\cdot)$ is included in the majority of mathematical software packages.

\section{B. Partial Relay Selection}

In this case, given that the relay selection is implemented according to (8) the pdf of $\hat{\gamma}_{S R_{k}}$ is given by

$$
f_{\hat{\gamma}_{S R_{k}}}(x)=N\left[F_{\hat{\gamma}_{S R}}(x)\right]^{N-1} f_{\hat{\gamma}_{S R}}(x) .
$$

Moreover, the pdf of $\hat{\gamma}_{R_{k} D}$ is the exponential distribution with average value $\bar{\gamma}_{R D}$, since the $R_{i}-D, i=1, \ldots, N$, links are not taken into account for relay selection. Consequently, $f_{\gamma_{S R_{k}}}(\cdot)$ is obtained by substituting (14) and (26) into (12), yielding

$$
f_{\gamma_{S R_{k}}}(x)=N \sum_{m=0}^{N-1} \frac{(-1)^{m}}{\bar{\gamma}_{S R}}\left(\begin{array}{c}
N-1 \\
m
\end{array}\right) \frac{e^{-\frac{(m+1) x}{\left(m\left(1-\rho_{1}^{2}\right)+1\right) \bar{\gamma} S R}}}{m\left(1-\rho_{1}^{2}\right)+1}
$$

while $F_{\gamma_{S R_{k}}}(\cdot)$ is given as

$$
F_{\gamma_{S R_{k}}}(x)=N \sum_{m=0}^{N-1} \frac{(-1)^{m}\left(\begin{array}{c}
N-1 \\
m
\end{array}\right)\left(1-e^{-\frac{(m+1)}{\left(m\left(1-\rho_{1}^{2}\right)+1\right) \bar{\gamma}_{S R}} x}\right)}{m+1} .
$$

Therefore, an expression for the outage probability is obtained from (10), in conjunction with (28), yielding

$$
\begin{aligned}
& F_{\gamma_{k}}\left(\gamma_{T}\right)=1-2 N \sum_{m=0}^{N-1}\left[(-1)^{m}\left(\begin{array}{c}
N-1 \\
m
\end{array}\right)\right. \\
& \times \sqrt{\frac{\gamma_{T}\left(\gamma_{T}+1\right)}{\bar{\gamma}_{R D} \bar{\gamma}_{S R}(m+1)\left[1+m\left(1-\rho_{1}^{2}\right)\right]}} \\
& \times e^{-\frac{\left[\bar{\gamma}_{R D}+\bar{\gamma}_{S R}+m\left(\bar{\gamma}_{R D}+\bar{\gamma}_{S R}-\bar{\gamma}_{S R} \rho_{1}^{2}\right)\right] \gamma_{T}}{\bar{\gamma}_{S R} \bar{\gamma}_{R D}\left[1+m\left(1-\rho_{1}^{2}\right)\right]}} \\
& \left.\times K_{1}\left(2 \sqrt{\frac{(m+1) \gamma_{T}\left(\gamma_{T}+1\right)}{\bar{\gamma}_{S R} \bar{\gamma}_{R D}\left[1+m\left(1-\rho_{1}^{2}\right)\right]}}\right)\right]
\end{aligned}
$$

\section{Numerical Results AND Discussion}

In this section, we illustrate the effects of outdated CSI on the outage performance of the best relay selection and partial relay selection schemes. It is noted that all curves provided in Figs. 2-4 were confirmed by simulations (not shown here).

In Fig. 2 the outage probability of best relay selection for different correlation coefficients $\rho_{1}$ and $\rho_{2}$ is depicted versus the normalized average SNR of the $S-R$ and $R-D$ links; the normalization is with respect to the outage threshold SNR, $\gamma_{T}$, which is our examples equals unity. The number of available relays is $N=3$, while all participating links are assumed to be symmetrical, so that $\bar{\gamma}_{S R_{i}}=\bar{\gamma}_{R_{i} D}, i=1,2,3$. The main result extracted from Fig. 2 is that outdated CSI at the $\mathrm{CU}$ significantly affects the outage performance of best relay selection, since the corresponding outage curves highly depend on the values of $\rho_{1}, \rho_{2}$. As expected, when either $\rho_{1}$ or $\rho_{2}$ deviate from unity the diversity order of best relay selection drops to one, whereas the diversity order equals the number of available relays in the perfect CSI case (i.e., $\rho_{1}=\rho_{2}=1$ ). One may note that this deviation from the perfect CSI case in terms of diversity order is evident even when these correlation coefficients take relatively high values, e.g., $\rho_{1}=\rho_{2}=0.95$. Using the Jakes' model in (3), we infer that $\rho_{1}=\rho_{2}=0.95$ corresponds to updating the channel estimates in a rate which is greater than or equal to $14 f_{d, S R}\left(=14 f_{d, R D}\right)$ which, for the typical case where a mobile transceiver operating at the frequency of $1.8 \mathrm{GHz}$ is moving at a speed of $50 \mathrm{~km} / \mathrm{h}$, corresponds to a time interval between consecutive estimations of about $700 \mu \mathrm{sec}$. We further note that, due to symmetry, the 


$$
\begin{aligned}
& F_{\gamma_{k}}\left(\gamma_{T}\right)=N \sum_{n=0}^{N-1} \frac{\left(\exp \left(-\frac{\left(\bar{\gamma}_{S R}+\bar{\gamma}_{R D}\right)(n+1) \gamma_{T}}{\bar{\gamma}_{R D}\left(\bar{\gamma}_{S R}\left(1+n\left(1-\rho_{2}^{2}\right)\right)+\bar{\gamma}_{R D}(1+n)\left(1-\rho_{2}^{2}\right)\right)}\right)-1\right) n \bar{\gamma}_{S R}+e^{-\frac{\gamma_{T}}{\bar{\gamma}_{R D}}\left(1-e^{\frac{\gamma_{T}}{\bar{\gamma}_{R D}}}\right)(n+1) \bar{\gamma}_{R D}}}{\left[(-1)^{n+1}\left(\begin{array}{c}
N-1 \\
n
\end{array}\right)\right]^{-1}(n+1)\left(\bar{\gamma}_{R D}+n \bar{\gamma}_{S R}+n \bar{\gamma}_{R D}\right)} \\
& +N^{2} \sum_{n=0}^{N-1} \sum_{m=0}^{N-1} \frac{(-1)^{n+m}\left(\begin{array}{c}
N-1 \\
m
\end{array}\right)\left(\begin{array}{c}
N-1 \\
n
\end{array}\right)}{\left(\bar{\gamma}_{R D}+m \bar{\gamma}_{S R}+m \bar{\gamma}_{R D}\right)\left(\bar{\gamma}_{S R}+n \bar{\gamma}_{S R}+n \bar{\gamma}_{R D}\right)}\left\{e ^ { - \frac { \gamma _ { T } } { \overline { \gamma } } } \overline { \gamma } _ { S R } \left[e^{\frac{\gamma_{T}}{\bar{\gamma}_{S R}} \bar{\gamma}_{R D}-2 \sqrt{\frac{\bar{\gamma}_{R D} \gamma_{T}\left(\gamma_{T}+1\right)}{\bar{\gamma}_{S R}}}}\right.\right. \\
& \left.\times K_{1}\left(2 \sqrt{\frac{\gamma_{T}\left(\gamma_{T}+1\right)}{\bar{\gamma}_{S R} \bar{\gamma}_{R D}}}\right)\right]+\frac{n \bar{\gamma}_{R D} e^{-\frac{\gamma_{T}}{\bar{\gamma}_{R D}}}}{(n+1)}\left[\bar{\gamma}_{R D}-2 \exp \left(-\frac{\left(\bar{\gamma}_{S R}+\bar{\gamma}_{R D}\right)(n+1) \gamma_{T}}{\bar{\gamma}_{S R}\left(\bar{\gamma}_{R D}\left(1+n\left(1-\rho_{1}^{2}\right)\right)+\bar{\gamma}_{S R}(1+n)\left(1-\rho_{1}^{2}\right)\right)}\right)\right. \\
& \left.\times \sqrt{\frac{\bar{\gamma}_{R D}\left(\gamma_{T}+1\right)\left(\bar{\gamma}_{S R}+\bar{\gamma}_{R D}\right)(n+1) \gamma_{T}}{\bar{\gamma}_{S R}\left(\bar{\gamma}_{R D}\left(1+n\left(1-\rho_{1}^{2}\right)\right)+\bar{\gamma}_{S R}(1+n)\left(1-\rho_{1}^{2}\right)\right)}} K_{1}\left(\sqrt{\frac{4 \bar{\gamma}_{R D}\left(\gamma_{T}+1\right)\left(\bar{\gamma}_{S R}+\bar{\gamma}_{R D}\right)(n+1) \gamma_{T}}{\bar{\gamma}_{S R}\left(\bar{\gamma}_{R D}\left(1+n\left(1-\rho_{1}^{2}\right)\right)+\bar{\gamma}_{S R}(1+n)\left(1-\rho_{1}^{2}\right)\right)}}\right)\right] \\
& +m \bar{\gamma}_{S R} \exp \left(-\frac{\gamma_{T}\left[\bar{\gamma}_{S R}^{2}(m+1)+\bar{\gamma}_{S R} \bar{\gamma}_{R D}\left(2+m\left(2-\rho_{2}^{2}\right)\right)+\bar{\gamma}_{R D}^{2}(m+1)\left(1-\rho_{2}^{2}\right)\right]}{\bar{\gamma}_{S R} \bar{\gamma}_{R D}\left[\bar{\gamma}_{S R}\left(1+m\left(1-\rho_{2}^{2}\right)\right)+\bar{\gamma}_{R D}(1+m)\left(1-\rho_{2}^{2}\right)\right]}\right) \\
& \times\left[\bar{\gamma}_{S R} e^{\frac{\gamma_{T}}{\bar{\gamma}_{S R}}}-\frac{2 \sqrt{\gamma_{T}\left(\gamma_{T}+1\right) \bar{\gamma}_{S R}\left(\bar{\gamma}_{S R}+\bar{\gamma}_{R D}\right)(m+1)} K_{1}\left(2 \sqrt{\frac{\left(\bar{\gamma}_{S R}+\bar{\gamma}_{R D}\right)(m+1) \gamma_{T}\left(\gamma_{T}+1\right)}{\bar{\gamma}_{S R} \bar{\gamma}_{R D}\left[\bar{\gamma}_{S R}\left(1+m\left(1-\rho_{2}^{2}\right)\right)+\bar{\gamma}_{R D}(1+m)\left(1-\rho_{2}^{2}\right)\right]}}\right)}{\sqrt{\bar{\gamma}_{R D}\left[\bar{\gamma}_{S R}\left(1+m\left(1-\rho_{2}^{2}\right)\right)+\bar{\gamma}_{R D}(m+1)\left(1-\rho_{2}^{2}\right)\right]}}\right] \\
& +\frac{m n \bar{\gamma}_{S R}\left(\bar{\gamma}_{S R}+\bar{\gamma}_{R D}\right)}{n+1}\left[\frac{\bar{\gamma}_{R D} \exp \left(-\frac{\left(\bar{\gamma}_{S R}+\bar{\gamma}_{R D}\right)(m+1) \gamma_{T}}{\bar{\gamma}_{R D}\left(\bar{\gamma}_{S R}\left(1+m\left(1-\rho_{2}^{2}\right)\right)+\bar{\gamma}_{R D}(1+m)\left(1-\rho_{2}^{2}\right)\right)}\right)}{\left(\bar{\gamma}_{S R}+\bar{\gamma}_{R D}\right)(m+1)}\right. \\
& +\frac{\exp \left(-\frac{\left(\bar{\gamma}_{S R}+\bar{\gamma}_{R D}\right) \gamma_{T}\left[\bar{\gamma}_{S R}^{2}(m+1)(n+1)\left(1-\rho_{1}^{2}\right)+\bar{\gamma}_{R D}^{2}(m+1)(n+1)\left(1-\rho_{2}^{2}\right)+\bar{\gamma}_{S R} \bar{\gamma}_{R D}\left(2+n\left(2-\rho_{1}^{2}\right)+m\left(2-\rho_{2}^{2}+n\left(2-\rho_{1}^{2}-\rho_{2}^{2}\right)\right)\right)\right]}{\bar{\gamma}_{S R} \bar{\gamma}_{R D}\left[\bar{\gamma}_{R D}\left(1+n\left(1-\rho_{1}^{2}\right)\right)+\bar{\gamma}_{S R}(n+1)\left(1-\rho_{1}^{2}\right)\right]\left[\bar{\gamma}_{S R}\left(1+m\left(1-\rho_{2}^{2}\right)\right)+\bar{\gamma}_{R D}(m+1)\left(1-\rho_{2}^{2}\right)\right]}\right)}{} \\
& \sqrt{\bar{\gamma}_{S R}(m+1)} \\
& \times 2 \sqrt{\frac{\bar{\gamma}_{R D} \gamma_{T}\left(\gamma_{T}+1\right)(n+1)\left[\bar{\gamma}_{S R}\left(1+m\left(1-\rho_{2}^{2}\right)\right)+\bar{\gamma}_{R D}(m+1)\left(1-\rho_{2}^{2}\right)\right]}{\bar{\gamma}_{R D}\left(1+n\left(1-\rho_{1}^{2}\right)\right)+\bar{\gamma}_{S R}(n+1)\left(1-\rho_{1}^{2}\right)}} \\
& \left.\left.\times K_{1}\left(\sqrt{\frac{4 \bar{\gamma}_{S R} \bar{\gamma}_{R D}(m+1)(n+1) \gamma_{T}\left(\gamma_{T}+1\right)}{\bar{\gamma}^{2}\left[\bar{\gamma}_{R D}\left(1+n\left(1-\rho_{1}^{2}\right)\right)+\bar{\gamma}_{S R}(n+1)\left(1-\rho_{1}^{2}\right)\right]\left[\bar{\gamma}_{S R}\left(1+m\left(1-\rho_{2}^{2}\right)\right)+\bar{\gamma}_{R D}(m+1)\left(1-\rho_{2}^{2}\right)\right]}}\right)\right]\right\}
\end{aligned}
$$

effect of outdated estimates for the $S-R_{i}$ links on the overall outage performance is identical to that of the $R_{i}-D$ links, so that the outage curves of the cases where $\left(\rho_{1}, \rho_{2}\right)=(0.5,1)$ and $\left(\rho_{1}, \rho_{2}\right)=(1,0.707)$ coincide with the $\left(\rho_{1}, \rho_{2}\right)=(1,0.5)$ and $\left(\rho_{1}, \rho_{2}\right)=(0.707,1)$ curves, which are shown in Fig. 2 .

Similar conclusions regarding the performance of partial relay selection under various levels of outdated CSI can be extracted from Fig. 3. Nevertheless, one may observe that the diversity order of partial relay selection is equal to unity, regardless of the value of $\rho_{1}$. This is due to the intrinsic drawback of partial relay selection in terms of diversity order, as compared to the best relay selection scheme, where the lack of CSI knowledge of either the $S-R$ or the $R-D$ link renders partial relay selection incapable of taking full advantage of the inherent diversity potential. To this end, it is pointed out that although in the best relay selection case any slight deviation from perfect CSI knowledge results in a significant outage performance degradation, the partial relay selection outage probability is affected to a much lesser extend when $\rho_{1} \neq 1$. As a cross check, one may notice from Figs. 2 and 3 that the outage performance of best relay selection with $\rho_{1}=\rho_{2}=0$ coincides with that of partial relay selection with $\rho_{1}=0$.

Interesting results regarding the relative outage performances of best relay selection and partial relay selection are derived from Fig. 4. In Fig. 4, the outage probabilities of the above schemes are plotted versus $\rho_{2}$, which represents the correlation coefficient between the actual channel gain of the $R_{i}-D$ links and the corresponding channel estimate, assuming $\gamma_{T}=1$ and $N=3$. Moreover, in Fig. 4 we have set $\rho_{1}=1$, implying that the $S-R_{i}$ channel estimates are perfectly updated, which may correspond to the downlink of the practical relaying application where fixed (infrastructure-based) relays are employed. It is also noted that similar results are obtained for $\rho_{1}<1$; however, those results are omitted here for brevity. In fact, we observe from Fig. 4 that there exists a crossing point between the outage performances of the best and the partial relay selection cases. This implies that depending on the level of CSI imperfection of the $R_{i}$-D links (or equivalently of the $S-R_{i}$ links if partial relay selection takes only the $R_{i}-D$ path into account), partial relay selection may outperform best relay selection in terms of 


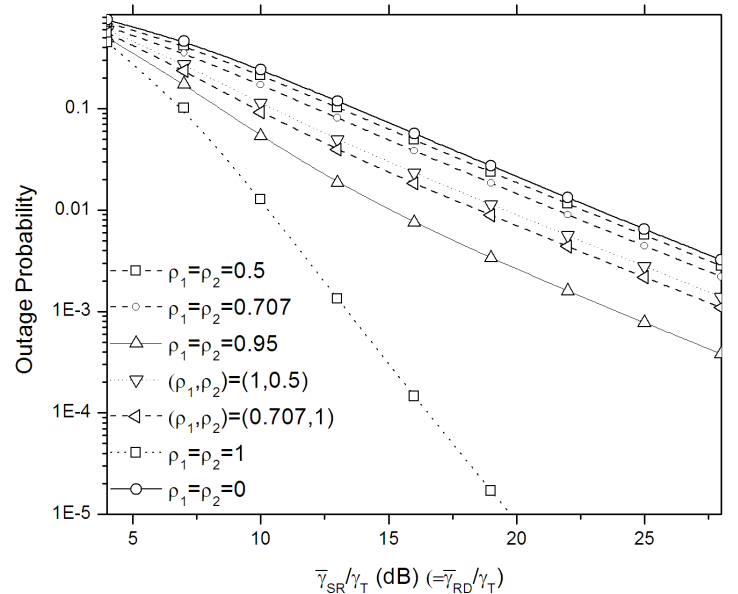

Fig. 2. Outage probability of the best relay selection scheme versus the normalized average SNR of the $S-R_{i}$ and $R_{i}-D$ links, for $N=3$.

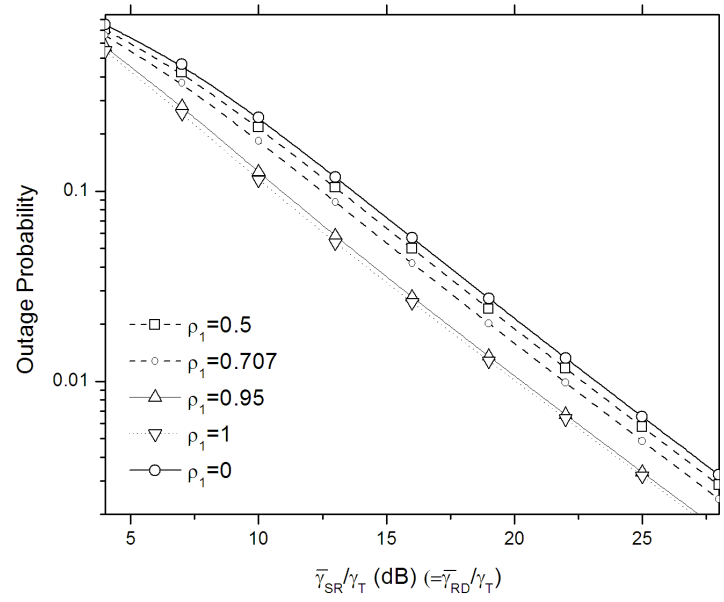

Fig. 3. Outage probability of the partial relay selection scheme versus the normalized average SNR of the $S-R_{i}$ and $R_{i}-D$ links, for $N=3$.

outage probability, and vice versa. To be more precise, since the $\rho_{2}$ parameter is defined as $\rho_{2}=J_{0}\left(2 \pi f_{d, R D} T_{d}\right)$, for the particular scenario where the value of $\rho_{1}$ is fixed the relative performances of best and partial relay selection are actually determined by the maximum Doppler frequency of the $R_{i}-D$ links. Therefore, from a system designer's perspective, given a maximum allowable value of channel estimation repetition rate, it is preferable to include the $R_{i}-D \mathrm{SNR}$ in the relay selection process only if the maximum Doppler frequency of the $R_{i}-D$ links is lower than a given threshold. This threshold is determined by the values of $\bar{\gamma}_{S R}$ and $\bar{\gamma}_{R D}$, through (3) and Fig. 4.

\section{REFERENCES}

[1] R. Pabst et al., "Relay-based deployment concepts for wireless and mobile broadband radio," IEEE Commun. Mag., vol. 42, no. 9, pp. 80-89, Sept.

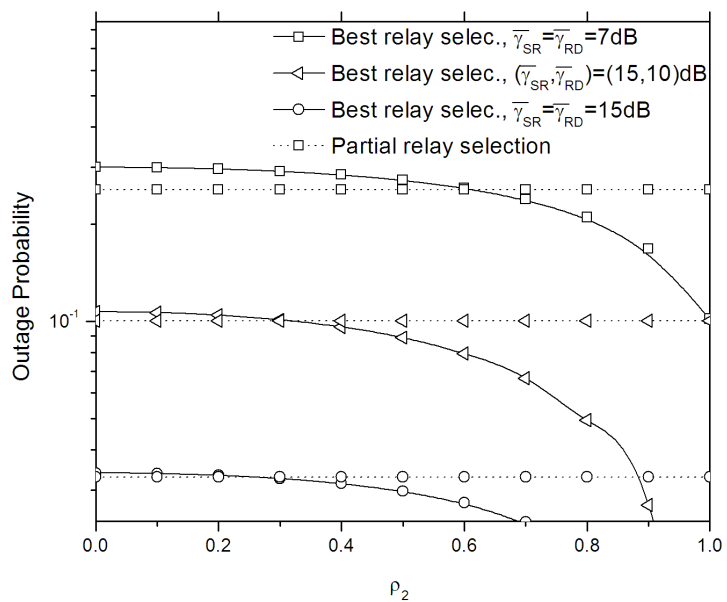

Fig. 4. Outage probability of best and partial relay selection versus $\rho_{2}$, for $\rho_{1}=1$ and $N=3$.

2004.

[2] Y. Yang, H. Hu, J. Xu, and G. Mao, "Relay technologies for WiMAX and LTE-Advanced mobile systems," IEEE Commun. Mag., vol. 47, pp. 100-105, Oct. 2009

[3] J. N. Laneman, D. N. C. Tse and G. W. Wornell, "Cooperative diversity in wireless networks: Efficient protocols and outage behavior," IEEE Trans. Inform. Theory, vol. 50, pp. 3062-3082, Dec. 2004.

[4] A. Bletsas, D. P. Reed, and A. Lippman, "A simple cooperative diversity method based on network path selection," IEEE J. Select. Areas Commun., vol. 24, pp. 659-672, Mar. 2006.

[5] I. Krikidis, J. S. Thompson, S. McLaughlin, and N. Goertz, "Amplify and forward with partial relay selection," IEEE Commun. Lett., vol. 12, pp. 235-237, Apr. 2008.

[6] E. Beres and R. Adve, "Selection Cooperation in Multi-Source Cooperative Networks," IEEE Trans. Wireless Commun., vol. 7, pp. 118-127, Jan 2008.

[7] Y. Jing and H. Jafarkhani, "Single and multiple relay selection schemes and their achievable diversity orders," IEEE Trans. Wireless Commun., vol. 8, pp. 1414-1423, Mar. 2009.

[8] S. Lee, M. Han , T. Yu, and D. Hong "Average SNR and ergodic capacity analysis for opportunistic DF relaying with outage over Rayleigh fading channels," IEEE Trans. Wireless Commun., vol 8, pp. 2008, Jun 2009.

[9] H. A. Suraweera, D. S. Michalopoulos, and G. K. Karagiannidis, "Semiblind amplify-and-forward with partial relay selection," Electron. Lett., vol. 45, pp. 317-318, Mar. 2009.

[10] D. S. Michalopoulos and G. K. Karagiannidis, "Performance analysis of single relay selection in Rayleigh fading," IEEE Trans. Wireless Commun., vol. 7, pp. 3718-3724, Oct. 2008.

[11] Y. Zhao, R. Adve, and T. J. Lim, "Symbol error rate of selection amplifyand-forward relay systems," IEEE Commun. Lett., vol. 10, pp. 757-759, Nov. 2006.

[12] J. L. Vicario, A. Bel, J. A. Lopez-Salcedo, and G. Seco, “Opportunistic relay selection with outdated CSI: Outage probability and diversity analysis," IEEE Trans. Wireless Commun., vol. 8, pp. 2872-2876, June 2009.

[13] Y. Ma, D. Zhang, A Leith, and Z. Wang "Error performance of transmit beamforming with delayed and limited feedback," IEEE Trans. Wireless Commun., vol. 8, pp. 1164-1170, March 2009.

[14] I. S. Gradshteyn and I. M. Ryzhik, Table of Integrals, Series and Products, 7th ed., New York: Academic, 2007.

[15] H. A. David and H. N. Nagaraja, Order Statistics. 3rd ed. New York: Wiley, 2003. 